\title{
Surveillance study of apparent life-threatening events (ALTE) in the Netherlands
}

\author{
Ben A. Semmekrot • Bregje E. van Sleuwen • Adele C. Engelberts • \\ Koen F. M. Joosten • Jaap C. Mulder $\cdot$ K. Djien Liem • Rob Rodrigues Pereira • \\ Rob P. G. M. Bijlmer • Monique P. L'Hoir
}

Received: 3 May 2009 / Accepted: 8 June 2009 /Published online: 21 June 2009

(C) The Author(s) 2009. This article is published with open access at Springerlink.com

\begin{abstract}
SIDS and ALTE are different entities that somehow show some similarities. Both constitute heterogeneous conditions. The Netherlands is a low-incidence country for SIDS. To study whether the same would hold for ALTE, we studied the incidence, etiology, and current treatment of ALTE in The Netherlands. Using the Dutch Pediatric Surveillance Unit, pediatricians working in second- and third-level hospitals in the Netherlands were asked to report any case of ALTE presented in their hospital from January 2002 to January 2003. A questionnaire was
\end{abstract}

\footnotetext{
B. A. Semmekrot $(\square)$

Department of Pediatrics, Canisius-Wilhelmina Hospital, P. O. Box 9015, 6500 GS Nijmegen, The Netherlands e-mail: b.semmekrot@cwz.nl

B. E. van Sleuwen · R. Rodrigues Pereira - M. P. L'Hoir Netherlands Pediatric Surveillance Unit, TNO Quality of Life, Prevention and Health,

P. O. Box 2215, 2301 CE Leiden, The Netherlands

A. C. Engelberts

Department of Pediatrics, Orbis Medical Centre,

P. O. Box 5500, $6130 \mathrm{MB}$ Sittard, The Netherlands

K. F. M. Joosten

Erasmus Medical Centre, Sophia Children's Hospital,

P. O. Box 2060, 3000 CB Rotterdam, The Netherlands

J. C. Mulder

Rijnstate Hospital,

P. O. Box 9555, 6800 TA Arnhem, The Netherlands

K. D. Liem

Department of Neonatology, Radboud University Medical Centre,

P. O. Box 9101, 6500 HB Nijmegen, The Netherlands

R. P. G. M. Bijlmer

Academic Medical Centre,

P.O. Box 22660, 1105 AZ Amsterdam, The Netherlands
}

subsequently sent to collect personal data, data on pregnancy and birth, condition preceding the incident, the incident itself, condition after the incident, investigations performed, monitoring or treatment initiated during admission, any diagnosis made at discharge, and treatment or parental support offered after discharge. A total of 115 cases of ALTE were reported, of which 110 questionnaires were filled in and returned (response rate 97\%). Based on the national birth rate of 200,000 , the incidence of ALTE amounted 0.58/1,000 live born infants. No deaths occurred. Clinical diagnoses could be assessed in $58.2 \%$. Most frequent diagnoses were (percentages of the total of 110 cases) gastro-esophageal reflux and respiratory tract infection (37.3\% and $8.2 \%$, respectively); main symptoms were change of color and muscle tone, choking, and gagging. The differences in diagnoses are heterogeneous. In 34\%, parents shook their infants, which is alarmingly high. Preand postmature infants were overrepresented in this survey (29.5\% and $8.2 \%$, respectively). Ten percent had recurrent ALTE. In total, $15.5 \%$ of the infants were discharged with a home monitor. In conclusion, ALTE has a low incidence in second- and third-level hospitals in the Netherlands. Parents should be systematically informed about the possible devastating effects of shaking an infant. Careful history taking and targeted additional investigations are of utmost importance.

Keywords Apparent life-threatening event (ALTE) · Sudden infant death syndrome (SIDS) - Infant · Incidence

\section{Introduction}

Infants with an apparent life-threatening event (ALTE) are presented to medical services because of a sudden 
unexpected incident, i.e., an acute and unexpected change in behavior that alarmed the caregiver and that was experienced by the caregiver as "life threatening". The initial episode may occur during sleep, wakefulness, or feeding and is most commonly described as a combination of several of the following: apnea, color change (cyanotic or pallid, occasionally erythematous), marked change in muscle tone (limpness, rarely rigidity), choking, or gagging $[13,15]$. In most cases, the observer reported that the episode was potentially life threatening and, sometimes, that the child had actually died and that a prompt intervention was associated with a normalization of the child's appearance, as defined at the ESPID meeting in Oslo, May 2003 [15].

The severity of an ALTE relies only on the history given by witnesses. When first examined, the infant may sometimes show persistent limpness, color change, or abnormal state $[8,13,15]$. In the absence of objective means to document the severity of the incident, history taking is the major tool in the approach of ALTE $[11-13,15,17]$.

Disorders related to ALTE are digestive, neurological, respiratory, cardiovascular, metabolic, or endocrine of nature. Other conditions have been described, such as unaccustomed prone sleeping, parental mental disorders, child abuse, neglect, or smothering [11-13, 15, 17].

The occurrence of an ALTE gives rise to many hospital admissions. It may require intensive care treatment and multidisciplinary follow-up. Uniformity in approach, regarding both the diagnostic and the treatment phase, should be at the basis of care for infants with ALTE [17, 21]. It should be a principal goal in any case of ALTE to reduce the anxiety, existing in parents or caregivers, in a realistic and adequate manner, by taking the incident seriously. This includes meticulous history taking and performance, if indicated, of an efficacious problem-oriented additional investigation in the infant, in order to find a reasonable explanation for the incident $[11,13,15,17]$.

In the Netherlands, a country in which the definition "near-miss for SIDS" was abandoned since 1986, no systematic studies on ALTE have been performed (National Consensus, 1986). The cot death incidence is extremely low in the Netherlands $(0.09 / 1,000$ live born infants) [6]. Although there seems to be no clear evidence for a relation between ALTE and SIDS, infants who have experienced an ALTE are at somewhat increased risk for SIDS. Therefore, the question arises as to whether or not the incidence of ALTE is also low in the Netherlands.

On the basis of retrospective data from referred ALTE cases in the mid-nineties, we estimated that yearly about 50 cases had been referred to third level, and 100 to second-level hospitals [1]. Together with 200 yearly reported collapse reactions (categorized from mild to severe), it was assessed that annually 1.8/1,000 live born infants in the Netherlands had been referred to hospital due to ALTE [23].
To study ALTE more exactly, the item was incorporated in a Dutch national surveillance study. The objectives were to estimate the incidence of ALTE presented to second- and third-level hospitals in the Netherlands, assess the etiology, and describe current practices regarding its management.

\section{Methods and patients}

\section{Method}

The system of the Pediatric Surveillance Unit (PSU) was used. Analogous to the British Pediatric Surveillance Unit, the Dutch system was set up in the early 1990s using the same methodology. The Dutch PSU is also a member of the International Network of Pediatric Surveillance Units. Pediatricians, working in third- and second-level hospitals in the Netherlands, are asked to report monthly on five to ten infrequent disorders. All pediatricians operating in the Netherlands participate in the system, mostly via one responsible pediatrician per department or hospital. In 2001, the response rate via the PSU system amounted $92 \%$ in second- and $83 \%$ in third-level hospitals (www.nvk. pedianet.nl).

Pediatricians were asked to report any case of ALTE, presented in their hospital, from January 2002 to January 2003. If an ALTE was reported, a questionnaire was sent to collect personal data, data on pregnancy and birth, condition preceding the incident, the incident itself, situation after the incident, investigations performed, monitoring or treatment initiated during admission, any diagnosis made at discharge, and treatment or parental support provided after discharge.

\section{Patients}

Any child from birth to its second birthday who had an episode frightening to the observer and characterized by some combination of apnea, change of color, marked change in muscle tone, choking, or gagging was included.

\section{Results}

Response

In total, 115 ALTE's were reported. Of 115 questionnaires, subsequently sent, 110 were filled out and returned, a response of $97 \%$. With 200,000 infants born annually, this suggests an incidence of $0.58 / 1,000$ live born infants. Adding to these, the yearly 200 collapse reactions after vaccinations, reported at the National Institute for Health and Environment, the 
incidence of ALTE in the Netherlands is estimated to be 1.57/ 1,000 live born infants. No ALTE-associated deaths occurred during the study period.

\section{Demographic data}

Infants with low birth weight and/or small for gestational age, as well as first-born infants, were overrepresented (Table 1). More ALTE infants were born after 42 weeks of gestation, as compared to the general population. The event occurred at a mean age of 11.4 (SD 13.5) weeks.

The incident

The majority of ALTE was observed by the parents: in $30 \%$ by the mother alone, in $7 \%$ the father alone, in $47 \%$ both parents. In $1 \%$, the general practitioner, and in $15 \%$, another person than the above-mentioned, was present. Most incidents (91\%) happened at home: 53 in supine, three in prone, and one in side position. In 48 cases, no answer was available.

The majority of symptoms observed by the parents during the $24 \mathrm{~h}$ preceding the incident was related to the digestive system: vomiting, infections, and feeding problems (Table 2). Symptoms reported shortly after the incident were variable, i.e., color difference (pale or blue/cyanotic), change of muscle tone (hypotonic), and breathing problems (Table 3). In 19 cases, the attending physician indicated that the infant had completely recovered after $5 \mathrm{~min}$, whereas in three infants, recovery took more than $5 \mathrm{~min}$. Since this question was presented as an open question, it does not provide complete information.
Actions undertaken by the observer of the incident are depicted in Table 4: 54\% of 110 infants were taken up, 34\% shaken, $12 \%$ had mouth-to-mouth breathing applied on them. In other cases, the infant was stimulated by tapping, squeezing, blowing, or holding the infant upside down. In $72 \%$, the parents/caregivers sought medical help within half an hour. At time of presentation, none of the infants needed intubation or other means of resuscitation. In 33 of 101 cases in which this question was answered, a doctor had been consulted preceding the incident: $12 \%$ (of 33 ) in the last $24 \mathrm{~h}, 42 \%$ (of 33 ) in the preceding week, and $46 \%$ within a month. In 17 cases, the consulted doctor was a pediatrician, in nine a general practitioner, in three a community health care doctor, and in four "another doctor".

In 15 of 110 infants (14\%), medication was prescribed before the incident, i.e., antibiotics (3\%), asthma medication ( $\beta$-mimetic and/or anti-inflammation: $4 \%$ ), caffeine $(1 \%)$, laxatives $(2 \%)$, diuretics $(1 \%)$, xylomethazolin nose spray $(2 \%)$, local antimycotics $(2 \%)$, local hydrocortisone crème $(1 \%)$, and cisapride $(1 \%)$.

\section{Diagnostic procedures}

Table 5 shows the diagnostic approach the infants were subjected to. Investigations performed in the majority of infants were complete WBC, temperature, glucose measurement, and ECG. Metabolic investigation was performed in $15.5 \%$ of cases. Polysomnography was done in only five $(4.6 \%)$ of infants. Of these, two were performed in the hospital of admission and three in a third-level hospital.
Table 1 Demographic data of ALTE cases

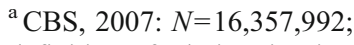
definition of ethnic minority: person of whom at least one of both parents not born in the Netherlands; data on gestational age are derived from the Netherlands Perinatal Registry, 2002

${ }^{\mathrm{b}}$ Seven percent of all infants in the Netherlands are born $<37$ weeks: $1.0 \%<32$ weeks; $3.5 \%$

32-36 weeks (data LNR)

${ }^{\mathrm{c}}$ Six Turkish parents, two North-African, two Surinamese and Dutch Antillian, two from "other European countries", two "unknown"

\begin{tabular}{lccc}
\hline & $N$ ALTE & ALTE (\%) & General population (\%) \\
\hline Boys & 110 & $62(56.4)$ & 51 \\
Birthweight $<2,500 \mathrm{~g}$ & 99 & $19(19.3)$ & 8 \\
Gestational age ${ }^{\mathrm{b}}$ & 110 & $11(10.0)$ & 1.6 \\
$<32$ weeks & & $21(19.5)$ & 6.3 \\
$32-37$ weeks & & $69(62.7)$ & 86.9 \\
$37-42$ weeks & & $9(8.2)$ & 5.2 \\
$\geq 42$ weeks & 104 & 11.4 & - \\
Age infants (weeks) & 99 & $51(51.5)$ & 47 \\
First-born infants & 66 & $5(7.6)$ & 2 \\
Maternal age at birth child & & $33(50.0)$ & 52 \\
$<20$ years & & $28(42.4)$ & 46 \\
$20-30$ years & 102 & $17(15.5)$ & 19.4 \\
$\geq 30$ years & 97 & $18(18.6)$ & - \\
Ethnic minority & & & - \\
Mother & & & \\
Father & & & \\
\hline
\end{tabular}


Table 2 Signs and symptoms before the incident ( $N=110$, multiple signs or symptoms may occur in each case)

\begin{tabular}{lc}
\hline Symptom & $N(\%)$ \\
\hline Excessive vomiting & $28(25.5)$ \\
Common cold & $31(28.2)$ \\
Feeding problems & $21(19.1)$ \\
Drowsy/in-alert & $8(7.3)$ \\
Fever & $8(7.3)$ \\
Vaccination & \\
$<24$ h before incident & $5(4.5)$ \\
$24-48$ h before incident & $1(0.9)$ \\
Apnea & $6(5.5)$ \\
Diminished growth & $4(3.6)$ \\
Excessive crying $^{\text {Various illness }}{ }^{\mathrm{a}}$ & $3(2.7)$ \\
Diverse & $5(4.5)$ \\
\hline
\end{tabular}

${ }^{\text {a }}$ Aortic coarctation, mild hemophilia A, conjunctivitis

${ }^{\mathrm{b}}$ Excessive weight gain and vomiting (Surinamese mother), warm/ sweaty, cow's milk allergy-related rectal blood loss, overstretching, bradycardia, Stickler syndrome

\section{Clinical diagnoses}

A somatic condition, assumed to have caused the incidence, was found in $58.2 \%$. In $28 \%$ of cases, the incident remained unexplained. For unknown reasons, $13.7 \%$ of respondents did not answer this question. By a number of closed questions, specific information was collected with respect to the cause of the incident (Table 6). No relation with a previous vaccination was mentioned (less than 24, 24-48, or more than $48 \mathrm{~h}$ ).

\section{Recurrent ALTE}

Ten percent of all infants had recurrent ALTE (reported by the parents); in six infants, this happened more than once.

Table 3 Condition of the infant shortly after the incident $(N=110)$

\begin{tabular}{lc}
\hline Condition & $N(\%)$ \\
\hline Pallor & $47(42.7)$ \\
Hypotonia & $67(60.9)$ \\
Blue/cyanotic & $41(37.3)$ \\
Breathing difficulties & $5(4.5)$ \\
Red & $10(9.1)$ \\
Hypertonic/overdistension/crying & $6(5.5)$ \\
Excessive mucus production & $5(4.5)$ \\
"Jerkin limbs" & $3(2.7)$ \\
Eye movements & $3(2.7)$ \\
Vomiting & $2(1.8)$ \\
\hline
\end{tabular}

Table 4 Actions undertaken by observer of the incident $(N=110)$

\begin{tabular}{lc}
\hline Action & $N(\%)$ \\
\hline Picked up the infant & $59(53.6)$ \\
Shook the baby & $37(33.6)$ \\
Squeezed hand/foot & $9(8.2)$ \\
Mouth-to-mouth breathing & $13(11.8)$ \\
Applied cold cloth & $3(2.7)$ \\
Chest compressions & $3(2.7)$ \\
Stimulated any other way & $43(39.1)$ \\
\hline
\end{tabular}

Earlier incidents had taken place five times during waking and six times (presumably) during sleep. Twenty-one cases recurred during admission.

\section{GER}

Significant acid reflux could be demonstrated in 31 from 53 infants $(58.5 \%)$ who underwent pH-metry. By contrast, in 22 of 53 infants (41.5\%), no acid gastro-esophageal reflux (GER) was detected. Of the 31 infants with significant GER, 27 (87\%) received medication, whereas 12 of GERproven infants (38.7\%) did not. Medications provided were cisapride (19), ranitidine (11), omeprazol (eight), and motilium (one).

\section{Treatment/parental support}

Ninety-six infants were subjected to in-hospital cardiorespiratory monitoring for relatively short periods: $5 \mathrm{~h}$ or less (three infants), $24 \mathrm{~h}$ (ten infants), $36 \mathrm{~h}$ (two infants), $48 \mathrm{~h}$

Table 5 Diagnostic approach/medical investigations performed $(N=110)$

\begin{tabular}{ll}
\hline Investigation & Percent of total \\
\hline Complete WBC & 82.7 \\
Temperature & 80.0 \\
Glucose & 70.0 \\
ECG & 61.8 \\
Chest X-ray & 49.1 \\
pH-metry & 48.2 \\
EEG & 35.8 \\
Respiratory syncytial virus & 23.6 \\
Metabolic investigation & 15.5 \\
Polysomnography & 4.5 \\
Blood gas analysis & 11.8 \\
Cerebral spinal fluid analysis & 2.7 \\
Cardiologic (other than ECG) & 2.0 \\
Pertussis diagnostics & 0.9 \\
\hline
\end{tabular}


Table 6 Diagnoses mentioned in the literature and results of present study (percentages of all causes) n.m. not mentioned

${ }^{a}$ All pertussis

${ }^{\mathrm{b}}$ All but two: RS virus

\begin{tabular}{lllc}
\hline Diagnosis & $\begin{array}{l}\text { Davies and Gupta [11] } \\
(N=65), \%\end{array}$ & $\begin{array}{l}\text { McGovern and Smith [17] } \\
(N=643), \%\end{array}$ & $\begin{array}{l}\text { Present study } \\
(N=110), \%\end{array}$ \\
\hline Relatively frequent diagnosis & & & 37.3 \\
GER & 26 & 31 & 0 \\
Seizures & 9 & 11 & 17.3 \\
Choking/gagging & n.m. & n.m. & $8.2^{\mathrm{b}}$ \\
Respiratory tract infection & $9^{\mathrm{a}}$ & 8 & 14.6 \\
Less frequent diagnosis & & & 2.7 \\
Apnea & 1.5 & $n . m$. & 0 \\
Cardiac & 3 & 0.8 & 1 \\
Metabolic & 1.5 & 1.5 & 0 \\
ENT & 0 & 3.6 & 1.8 \\
UIT & 8 & 0 & 0 \\
Breath-holding spells & $\mathrm{n} . \mathrm{m}$. & 2.3 & 8.2 \\
Factitious & 3 & 0.3 & 23 \\
Unknown & 23 & & \\
\hline
\end{tabular}

(36 infants), 1 week (20 infants), 2 weeks (15 infants), 3 weeks (five infants). Only five infants were monitored for a period longer than 3 weeks, the reason for which is unknown.

During their stay in the hospital, a change of feeding was applied in 25 infants.

Seventeen infants (15.5\%) were discharged with a home monitor: a cardiorespiratory monitor in 14 (12.7\%), apnea monitor in one, and "other type" monitor in two cases. In 72 (65.5\%), no home monitor was used, but 17 among them participated in a special home support program (so-called weighing scale program) analogous to the CONI program in the UK [21]. From those with a home monitor, five $(33.3 \%)$ were monitored for less than 3 months, three (20\%) between 3 and 6 months, two $(13.3 \%)$ in 9-12 months, and three (20\%) in 12 months or longer. From the other cases, no data on the period of monitoring are known. All parents of infants receiving some kind of home monitoring participated in a simple resuscitation course.

During their stay in the hospital, parents received help from a social worker in $15 \%$ and from a psychologist in $8 \%$ of cases. In five families, only one, and in the other cases, more sessions were needed.

At discharge, 38\% of infants had medications prescribed, including cisapride (16), ranitidine (ten), omeprazol (eight), motilium (three), antacid (one), antibiotics (two), lactulose (two), inhalation steroids (two), ipratropium p.i. (one), diuretics (one), fenobarbitone (one), diazepam rectiole (one), clobetason crème (one), ferro (one), and "expectorant" (one). After discharge, six parents were referred to a psychologist or social worker. Follow-up was realized in $89 \%$ of the cases. Of note, cisapride at present is no longer used in the Netherlands.
Family history and social context

Two families had lost another child, one due to hydrocephalus and one to trisomy 18. In five cases, the parents had experienced anxiety and/or stress, e.g., because of previous prenatal asphyxia or GER in an older child. In two families, epilepsy had been diagnosed in other children. In the grandparents' families, the following disorders had occurred: epilepsy (five infants), cot death (one), ALTE (one), and "sudden death" (two). Pediatricians' concerns with respect to the social environment were expressed to several families. In one family, the pediatrician perceived parental reactions as inadequate, in one an older child had been presented with ALTE (perceived by the pediatrician as socially alarming), one family was socially isolated, and one family had presented the infant to several hospitals. Four families had severe psychosocial problems.

\section{Discussion}

We present here a systematic study on the incidence of ALTE in the Netherlands. The study shows that ALTE represents a heterogeneous event with an inconsistent follow-up, the nature and severity of which shows wide variation.

More than half of the infants with ALTE (51.5\%) were first born. The reason for this high percentage is unknown. Davies and Gupta mention $34 \%$ as first born [11]. An explanation might be that parents are less confident with their first child and interpret situations more easily as life threatening. It cannot be excluded that part of the overrepresentation of first born, low birthweight, and/or small for gestational age infants is explained by the relatively small numbers in the study group. 
Our study suggests an incidence of 0.58/1,000 live born infants. This is comparable with the incidence of $0.6 / 1,000$ live born reported by McGovern and Smith [17] and with 0.46/1,000 live born infants, reported from the prospective 1984-1986 Sweden study [25], but much lower than that of $2.46 / 1,000$, recently reported from the prospective $1993-$ 2001 study from the province of Tyrol, Austria [16]. Interestingly, when we add the annual 200 collapse reactions after vaccinations, registered at the Dutch National Institute for Health and Environment, the incidence of ALTE in the Netherlands would be 1.57/1,000 live born infants, comparable with the incidence in Austria. For comparison, the mean annual admission rate for ALTE in New Zealand is 9.4/1,000 live births, during the period 1986-1994 [19]. The large difference between the incidence found in our study and that in New Zealand might be explained by underestimation of the Dutch numbers. Because of the heterogeneity of the ALTE group, conditions and severity differ strongly and may not be perceived seriously enough to warrant hospital referral. In the Netherlands, a rather conservative referral strategy exists. General practitioners always examine the children physically before referring them to the hospital, and only serious cases are brought to the hospital emergency department immediately.

Risk factors for ALTE, mentioned in the literature, are age more than 2 months [5, 6], recurrent ALTE [11], prematurity [6], and positive medical history [6]. The age of presentation of ALTE in our study was 11.4 weeks. Earlier studies mention 7 weeks [11] and 8.8 weeks [12].

The percentage of ALTE infants born prematurely $(<37$ weeks) was $29.5 \%$, significantly higher than the $13 \%$ from the general population (versus $7.4 \%$ in the control group), as reported by Kiechl-Kohlendorfer et al. [18] and the $15 \%$ (<34 weeks) reported by Davies and Gupta [11], but comparable with the $32.7 \%$ reported by De Piero et al. [12]. Consistent with earlier data [20], we found no relation between previous vaccination and ALTE in premature infants.

The percentage of ALTE infants born after 42 weeks of gestation was $8.2 \%$, significantly higher than the corresponding percentage in the general population. To our knowledge, this is the first time an increased number of postmature infants is noted in an ALTE population. It is possible, however, that the difference with the general population is caused by the relatively small numbers in the study group. If true, we have no clear explanation for this phenomenon, but psychological mechanisms such as high anxiety levels might be involved. Possible explaining factors such as asphyxia and meconium aspiration were not noted in the postmature infants in the present study.

Feeding problems and common cold are the most frequently mentioned symptoms before, whereas changes of color and muscle tone, choking, and gagging are the most frequently mentioned symptoms after the incident. This is consistent with earlier reports $[2,11,17,18]$.

Our study confirms recent observations that various investigations performed, except for $\mathrm{pH}$-metry, have a low likelihood of a positive result [12, 21]. Diagnoses, mentioned as most likely explanations for the incident, are GER, apnea, choking or gagging in relation to feeding, respiratory tract infections, and seizures [7, 11, 22]. The differences in diagnoses observed in earlier studies and ours confirm the heterogeneity of ALTE (Table 6).

An alarmingly high percentage of parents (34\%) shook their infant after the event. For comparison, in a study of parental detrimental actions after infant crying in infants aged 1-6 months, Reijneveld et al. demonstrated $5.6 \%$ of Dutch parents to have smothered, slapped, or shaken their infant at least once because of its crying [20]. Bonkowsky et al. described infants having suffered from ALTE to be at higher risk for subsequent child abuse and adverse neurological outcomes [4]. Support and follow-up of families are necessary [3]. In the Netherlands, a primary preventive approach is in preparation by informing all parents systematically about possible devastating effects of shaking an infant $[4,14]$.

Studies on ALTE like ours and others have drawbacks. From most studies, it is not clear whether diagnoses concern either admission or discharge diagnoses. Also, the likelihood of finding a diagnosis increases when one specifically asks for it. This stresses the importance of a thorough history taking. We advise to take a repeated and well-planned history using a checklist. Six of eight studies from the McGovern and Smith review [17], including that from Davies and Gupta [11], used an initial investigation protocol. De Piero et al. did not use an initial investigation protocol, but paid much attention to targeted history taking [12]. In the present study, a fixed initial protocol was not used since it was not available in the Netherlands then. Since untargeted diagnostic investigations often do not lead to satisfying explanations for the incident $[12,21]$, a stepwise diagnostic approach would be more appropriate, as suggested earlier [11, 17], including thorough history taking. Mild incidents could thus remain without additional investigation, whereas a positive history is followed by a targeted investigation. The importance of careful history taking and physical examination has recently been stressed [21]. A professional ALTE guideline from the Dutch Pediatric Association, based on careful history taking and targeted use of additional investigations, following an algorithm, has now been accomplished, implemented (www.nvk.pedianet.nl), and accepted for publication (www.ntvg.nl).

Diagnostic procedures do not invariably offer an explanation for the incident. In about $25 \%$ of cases with 
no somatic explanation, psychosomatic and stress-related explanations may be involved, such as insecurity about motherhood by having been neglected or sexually abused as a young girl. If the pediatrician perceives signals of psychosocial stress, referral to a clinical psychologist should follow. Since the severity of an ALTE incident in such cases relies on the history given by a witness, psychological or behavioral explanations should thoroughly be explored and separated from suspicion related to direct abuse.

Only a minority of ALTE (4.5\%) requires polysomnography. This is consistent with the general policy in our country that polysomnography and monitoring be used only in selective cases. Other authors suggest that polysomnography and home monitoring might be helpful for further diagnosis of ALTE in very severe cases [9]. Also, polysomnography and home monitoring could be used to identify and document ALTE, in addition to its value as a rescue device [10] or to identify premature infants at increased risk for ALTE [8]. At this moment, there is no consensus regarding home monitoring. It is our opinion that patients for polysomnography should be carefully selected and provided with a clear medical indication. Because of the low numbers, polysomnography should take place in a limited number of specialized centers, thus enabling such centers to maintain their experience. Polysomnography in the Netherlands is performed in two third-level hospitals.

All ALTE infants that presented to the hospital were clinically monitored for some time. It is debated whether all infants with ALTE need to be admitted. A study addressing this question in infants presenting to the emergency department of a tertiary care hospital found multiple ALTE's before presentation and age less than 28 days risk factors severe enough to warrant admission [7]. The authors suggest that infants older than 30 days having experienced a single ALTE may be discharged safely from the hospital after evaluation.

Recurrent ALTE's may be associated with exprematurity [12], metabolic disease [24], or child abuse [7].

Only $15.5 \%$ of infants from our study entered a homemonitoring program. Another $17 \%$ followed a support program characterized by regular weighing and home visits by a health care nurse and follow-up by the pediatrician and the general practitioner [16]. Decisions for receiving home monitoring or other support programs were led by the presence of clinical symptoms, specific history, identified condition, or disease with high risk of recurrent apnea or extreme parental anxiety. A psychosocial indication for home monitoring is sometimes given, but in such cases, participation in the weighing scale program may offer adequate support as well. Some authors advise the use of home monitoring to identify events in recurrent or very severe cases of ALTE [9] or to identify infants at increased risk of ALTE $[8,10]$.
Psychosocial care was provided in about $20 \%$ of cases, the importance of which is stressed by the fact that pediatricians noted psychosocial problems in $16 \%$ of all cases. The large number of unexplained ALTE cases urges closer evaluation of the parents' psychological condition, e.g., if they probably perceive stress problems with their baby. Further research on psychosocial parental care in infants with ALTE seems warranted.

\section{Conclusion}

ALTE is a heterogeneous condition with a low incidence in second- and third-level hospitals in the Netherlands. Compared with the general population, ALTE infants more often were pre- or postmature. The study confirms recent reports stressing the importance of careful history taking and targeted additional investigations. Time has come for an evidence- or experience-based logical stepwise approach, as to diagnosis and treatment of this condition. Support to and follow-up of families of ALTE infants are necessary.

Acknowledgment We greatly acknowledge the kind cooperation of all Dutch pediatricians in reporting ALTE and completing the questionnaires.

Conflict of interest The authors have no financial relationships with organizations, nor were they sponsored in any kind for this research project.

Open Access This article is distributed under the terms of the Creative Commons Attribution Noncommercial License which permits any noncommercial use, distribution, and reproduction in any medium, provided the original author(s) and source are credited.

\section{References}

1. Apparent Life-Threatening Events (1994) Abstracts of the 16th Congress of the Dutch Pediatric Association. Veldhoven, The Netherlands

2. Arens R, Gozal D, Williams JC et al (1993) Recurrent apparent life-threatening events during infancy: a manifestation of inborn errors of metabolism. J Pediatr 123:415-418

3. Barr RG, Rivara FP, Barr M et al (2009) Effectiveness of educational materials designed to change knowledge and behaviors regarding crying and Shaken-Baby Syndrome in mothers of newborns. Pediatrics 123:972-980

4. Bonkowsky JL, Guenther E, Filloux FM, Srivastava R (2008) Death, child abuse, and adverse neurological outcome of infants after an Apparent Life-Threatening Event. Pediatrics 122:125-131

5. Brand DA, Altman RL, Purtill K, Edwards KS (2005) Yield of diagnostic testing in infants who have had an apparent lifethreatening event. Pediatrics 115:885-893

6. Central Bureau for Statistics (CBS) (2006) Voorburg, The Netherlands 
7. Claudius I, Keens T (2007) Do all infants with Apparent LifeThreatening Events need to be admitted? Pediatrics 119:679-683

8. Côté A, Hum C, Brouillette RT et al (1998) Frequency and timing of recurrent events in infants using home cardiorespiratory monitors. J Pediatr 312:783-798

9. Côté A (2006) Home and hospital monitoring for ALTE. Paediatr Resp Rev 7S:S199-S201

10. Daniels H, Naulaers G, Deroost F, Devlieger H (1999) Polysomnography and home documented monitoring of cardiorespiratory pattern. Arch Dis Child 81:434-436

11. Davies F, Gupta R (2002) Apparent life threatening event in infants presenting tot an emergency department. Emerg Med J 19:11-16

12. De Piero AD, Teach SJ, Chamberlain JM (2004) ED evaluation of infants after an apparent life-threatening event. Am J Emerg Med 22:83-86

13. DeWolfe CC (2005) Apparent life-threatening event: a review. Pediatr Clin North Am 52:1127-1146

14. Dias MS, Smith K, deGuehery K et al (2005) Preventing abusive head trauma among infants and young children: a hospital-based, parent education program. Pediatrics 115:e470-e477

15. Kahn A (2004) Recommended clinical evaluation of infants with an apparent life-threatening event. Consensus document of the European Society for the Study and Prevention of Infant Death. Eur J Pediatr 163:108-115

16. Kiechl-Kohlendorfer U, Hof D, Pupp Peglow U et al (2004) Epidemiology of apparent life threatening events. Arch Dis Child 90:297-300
17. McGovern MC, Smith MBH (2004) Causes of apparent life threatening events in infants: a systematic review. Arch Dis Child 89:1043-1048

18. Mitchell EA, Thompson JMD (2001) Parental reported apnoea, admissions to hospital and sudden infant death syndrome. Acta Paediatr 90:417-422

19. Okada K, Miyako M, Honma S et al (2003) Discharge diagnoses in infants with apparent life-threatening event. Pediatrics International 45:560-563

20. Reijneveld SA, van der Wal MF, Brugman E et al (2004) Infant crying and abuse. Lancet 364:1340-1342

21. Shah S, Sharieff GQ (2007) An update on the approach to apparent life-threatening events. Curr Opin Pediatr 19:288294

22. Slack MH, Schapira D (1999) Severe apnoeas following immunisation in premature infants. Arch Dis Child Fetal Neonatal Ed 81:F67-F68

23. Vermeer-de Bondt PE, Dzaferagic A, Phaff TAJ et al (2005) Adverse events following immunization under the National Vaccination Program of the Netherlands Number XI- reports in 2004. Bilthoven, The Netherlands (RIVM report 240071002)

24. Waite A, McKenzie A, Carpenter RG et al (1998) Report on 5000 babies using the CONI (Care of Next Infant) program. Foundation for the Study of Infant Deaths, London

25. Wennergren G, Milerad J, Lagercrantz H et al (1987) The epidemiology of sudden infant death syndrome and attacks of lifelessness in Sweden. Acta Paediatr Scand 76:898-906 